\title{
Frequency Diverse Array MIMO Radar Adaptive Beamforming with Range-Dependent Interference Suppression in Target Localization
}

\author{
Kuandong Gao, Huaizong Shao, Jingye Cai, Hui Chen, and Wen-Qin Wang \\ School of Communication and Information Engineering, University of Electronic Science and Technology of China, \\ Qingshuihexiaoqu, No. 2006 Xiyuan Road, Gaoxin, Xiqu, Chengdu, Sichuan 611731, China \\ Correspondence should be addressed to Kuandong Gao; kuandonggao@gmail.com
}

Received 1 February 2015; Accepted 10 September 2015

Academic Editor: Jaume Anguera

Copyright (C) 2015 Kuandong Gao et al. This is an open access article distributed under the Creative Commons Attribution License, which permits unrestricted use, distribution, and reproduction in any medium, provided the original work is properly cited.

\begin{abstract}
Conventional multiple-input and multiple-output (MIMO) radar is a flexible technique which enjoys the advantages of phasedarray radar without sacrificing its main advantages. However, due to its range-independent directivity, MIMO radar cannot mitigate nondesirable range-dependent interferences. In this paper, we propose a range-dependent interference suppression approach via frequency diverse array (FDA) MIMO radar, which offers a beamforming-based solution to suppress range-dependent interferences and thus yields much better DOA estimation performance than conventional MIMO radar. More importantly, the interferences located at the same angle but different ranges can be effectively suppressed by the range-dependent beamforming, which cannot be achieved by conventional MIMO radar. The beamforming performance as compared to conventional MIMO radar is examined by analyzing the signal-to-interference-plus-noise ratio (SINR). The Cramér-Rao lower bound (CRLB) is also derived. Numerical results show that the proposed method can efficiently suppress range-dependent interferences and identify range-dependent targets. It is particularly useful in suppressing the undesired strong interferences with equal angle of the desired targets.
\end{abstract}

\section{Introduction}

Multiple-input and multiple-output (MIMO) radar has received much attention in recent years. But for conventional colocated MIMO radar, the array manifold caused by time delay only depends on the angle and thus has difficulty in distinguishing the targets and interferences that have the same angle but different ranges by general beamforming techniques [1-3]. Moreover, the localization performance will also be significantly degraded $[4,5]$ with the targets with the same angle but different ranges. The conventional MIMO radar cannot distinguish range-dependent targets. According to the radar ambiguity function, the range and velocity of a target cannot be simultaneously estimated. Several methods have been suggested to solve these problems. A potential solution is using multiple distributed receivers. But it requires clock synchronization due to separated receivers, which is a technical challenge.

Frequency diverse array (FDA) radar proposed in [6-8] can suppress range-dependent interferences. Different from phased-array radar, a small frequency increment, as compared to the carrier frequency, is applied between FDA elements $[9,10]$. This small frequency increment results in a range-angle-dependent beampattern [11-13]. The time and angle periodicity of FDA beampattern was analyzed in [14]. A linear FDA was proposed in [15] for forward-looking radar ground moving target indication. The application of FDA for bistatic radar system was analyzed in [16]. And the imaging of FDA radar was investigated in [17-19]. In [20], we have derived the FDA Cramér-Rao lower bounds (CRLBs) for estimating direction, range, and velocity. Although FDA has drawn much attention in antenna and radar areas, the existing literature concentrates on FDA conceptual system design $[21,22]$. Furthermore, most of the FDA MIMO radar literature focuses on beamforming algorithm [23-25], and little work on target localization application and its performance analysis has been reported [26, 27].

In this paper, we design the FDA MIMO radar for rangedependent beamforming and target localization. Our proposed approach exploits jointly the advantages of FDA and 
MIMO techniques. The advantages of FDA MIMO radar as compared to conventional MIMO radar are investigated. Furthermore, the FDA MIMO radar performance is evaluated by the corresponding transmitting-receiving beampattern, and output signal-to-interference-plus-noise ratio (SINR) is also examined. The contributions of this paper can be summarized as follows. (i) We formulate the data model of FDA MIMO radar. (ii) Range-dependent interferences/targets with the same angle of the targets are suppressed/detected by the FDA MIMO adaptive beamformer. (iii) The CRLB of FDA MIMO radar is derived, along with extensive numerical results. (iv) We derive and verify the resolution probability of FDA MIMO radar localization.

The rest of this paper is organized as follows. In Section 2, we formulate the FDA MIMO radar data model. And we present an FDA MIMO radar adaptive beamforming approach with range-dependent interference suppression in Section 3. In Section 4, the multiple signal classification (MUSIC) algorithm is employed for target localization in angle-range dimension. Simulation results are provided in Section 5. Finally, conclusions are drawn in Section 6.

\section{Data Model of FDA MIMO Radar}

Consider an FDA MIMO radar equipped with $M$ colocated transmitting elements and $N$ colocated receiving elements. Assume the transmit and receiving arrays are closely located, so that a target located in far field can be seen by both of them at the same spatial angle. Each transmitting element sends out a distinct omnidirectional waveform $s_{m}(l), m=1,2,3, \ldots, M$ and $l=1,2,3, \ldots, L$. Let $\mathbf{s}(l)$ be the vector collecting all these waveforms. The baseband equivalent model, in complexvalued form, of the transmitted signals from the $m$ th transmit element can be expressed as

$$
a_{m}(\theta, r) s_{m}(l)
$$

where

$$
\begin{aligned}
a_{m} & (\theta, r) \\
& =e^{j * 2 \pi\left((m-1)(d / \lambda) \sin \theta-(m-1)(\Delta f / c) r+(m-1)^{2}(\Delta f / c) d \sin \theta\right)} \\
& \approx e^{j * 2 \pi((m-1)((d / \lambda) \sin \theta-(\Delta f / c) r))}
\end{aligned}
$$

is the $m$ th entry of the transmit steering vector with $\Delta f$ being the frequency increment [25] while $d$ is the element space, $\lambda$ is the carrier wavelength, $c$ denotes the light speed, and $\theta$ and $r$ are the target angle and range, respectively. As a comparison, (3) gives the $m$ th entry of the steering vector for a phased array, which is not related to the range variable $r$ :

$$
e^{(j 2 \pi(m-1)(d / \lambda) \sin \theta)} .
$$

The FDA MIMO radar transmit steering vector depends on both the range and the angle, whereas that of conventional MIMO radar depends only on the angle. Therefore, the interferences with the same angle of the targets cannot be suppressed by conventional MIMO radar but may be resolved by the FDA MIMO radar.
For a position with angle $\theta$ and range $r$, the phase difference between the two signals transmitted by two adjacent elements is

$$
\phi_{1}=2 \pi\left(\frac{d}{\lambda} \sin \theta-\frac{\Delta f}{c} r\right) .
$$

Thereby, the transmit steering vector is given by

$$
\mathbf{a}_{t t}(\theta, r)=\left[1, e^{j \phi_{1}}, e^{j 2 \phi_{1}}, \ldots, e^{j(M-1) \phi_{1}}\right]^{T} .
$$

After being reflected by the targets or interferences, the signals are received by the colocated receiving array. Taking the first element as a reference, the phase difference of the signals received by two adjacent elements is

$$
\varphi=2 \pi \frac{d}{\lambda} \sin \theta
$$

Similarly, the receiving steering vector is

$$
\mathbf{a}_{r}(\theta)=\left[1, e^{j \varphi}, e^{j 2 \varphi}, \ldots, e^{j(N-1) \varphi}\right]^{T},
$$

where $[\cdot]^{T}$ denotes the transpose operator. The phase difference $\phi_{1}$ of the transmit steering vector becomes

$$
\phi_{2}=2 \pi\left(\frac{d}{\lambda} \sin \theta-\frac{\Delta f}{c} 2 r\right)
$$

where $2 r$ is caused by reflected range. Therefore, the new transmit steering vector at the receiving array is given by

$$
\mathbf{a}_{t r}(\theta, r)=\left[1, e^{j \phi_{2}}, e^{j 2 \phi_{2}}, \ldots, e^{j(M-1) \phi_{2}}\right]^{T} .
$$

Suppose there is a target located at the position $\left(\theta_{0}, r_{0}\right)$ along with multiple interferences located at $\left(\theta_{i}, r_{i}\right) . N \times 1$ receiving complex vector of the receiver observations can be written as

$$
\begin{aligned}
\mathbf{x}(l)= & \delta_{0}(\tau) \mathbf{a}_{r}\left(\theta_{0}\right) \mathbf{a}_{t r}^{T}\left(\theta_{0}, r_{0}\right) \mathbf{s}(l) \\
& +\sum_{i} \delta_{i}(\tau) \mathbf{a}_{r}\left(\theta_{i}\right) \mathbf{a}_{t r}^{T}\left(\theta_{i}, r_{i}\right) \mathbf{s}(l)+\mathbf{n}(l), \\
& \quad l=1,2, \ldots, L,
\end{aligned}
$$

where $\delta_{0}(\tau)$ stands for the complex amplitude of the target and $\delta_{i}(\tau)$ represents the complex amplitude of the $i$ th interference source and $\mathbf{n}(l)$ is $N \times 1$ additive zero-mean white Gaussian noise term with covariance matrix $\sigma^{2} \mathbf{I}$, with $\sigma^{2}$ being the noise power. Note that $\tau$ is the slow time and $l$ is the fast time. Since $s_{m}(l)$ is orthogonal, the received signals can be processed by a matched filter, which outputs an $M \times N$ matrix:

$$
\begin{aligned}
\mathbf{Y}(l)= & {\left[\sum_{l=1}^{L} \mathbf{x}(l) \mathbf{s}^{H}(l)\right]\left[\sum_{l=1}^{L} \mathbf{s}(l) \mathbf{s}^{H}(l)\right]^{-1} } \\
= & \delta_{0}(\tau) \mathbf{a}_{r}\left(\theta_{0}\right) \mathbf{a}_{t r}^{T}\left(\theta_{0}, r_{0}\right) \\
& +\sum_{i} \delta_{i}(\tau) \mathbf{a}_{r}\left(\theta_{i}\right) \mathbf{a}_{t r}^{T}\left(\theta_{i}, r_{i}\right)+\mathbf{N}
\end{aligned}
$$


where

$$
\mathbf{N}=\left[\sum_{l=1}^{L} \mathbf{n}(l) \mathbf{s}^{H}(l)\right]\left[\sum_{l=1}^{L} \mathbf{s}(l) \mathbf{s}^{H}(l)\right]^{-1} .
$$

$[\cdot]^{-1}$ and $[\cdot]^{H}$ denote the matrix inverse operator and conjugate transpose operator, respectively, and $\sum$ denotes summation operator. Furthermore, it is easy to show that $\mathbf{N}$ is independent and identically distributed (i.i.d.) Gaussian entries with zero mean and variance $\sigma^{2}$. Stacking the columns of $\mathbf{Y}$, we obtain an $M N \times 1$ virtual data vector

$$
\mathbf{y} \triangleq \operatorname{vec}(\mathbf{Y})=\delta_{0}(\tau) \mathbf{a}\left(\theta_{0}, r_{0}\right)+\sum_{i} \delta_{i}(\tau) \mathbf{a}\left(\theta_{i}, r_{i}\right)+\mathbf{n},
$$

where vec denotes vectorization operator and $\mathbf{n} \triangleq \operatorname{vec}(\mathbf{N})$, and

$$
\mathbf{a}(\theta, r) \triangleq \mathbf{a}_{t r}(\theta, r) \otimes \mathbf{a}_{r}(\theta)
$$

denotes the joint transmit-receiving virtual steering vector, in which $\otimes$ stands for the Kronecker product. The interferenceplus-noise covariance matrix can be expressed as

$$
\mathbf{R}=\sum_{i} \alpha_{i}^{2} \mathbf{a}\left(\theta_{i}, r_{i}\right) \mathbf{a}^{H}\left(\theta_{i}, r_{i}\right)+\sigma^{2} \mathbf{I},
$$

where $i$ denotes the index of interferences and $\alpha_{i}^{2}=$ $E\left[\delta_{i}(\tau) \delta_{i}(\tau)^{*}\right]$ is the averaged power.

\section{Adaptive Beamforming and SINR Analysis for FDA MIMO Radar}

In this section, we consider the adaptive beamforming which can suppress the range-dependent interferences having the same angle but different ranges from that of targets. The performance is examined in terms of the output signal-tointerference-plus-noise ratio (SINR). For discussion convenience, we rewrite the output of matched filter as follows:

$$
\mathbf{y} \triangleq \operatorname{vec}(\mathbf{Y})=\delta_{0}(\tau) \mathbf{a}\left(\theta_{0}, r_{0}\right)+\sum_{i} \delta_{i}(\tau) \mathbf{a}\left(\theta_{i}, r_{i}\right)+\mathbf{n} .
$$

To suppress the interferences, the conventional MVDR beamformer for a target located at $\left(\theta_{0}, r_{0}\right)$ is employed by

$$
\begin{aligned}
\mathbf{w}_{\mathrm{MVDR}}=\underset{\mathbf{w}}{\arg \min } & \left(\mathbf{w}^{H} \mathbf{R w}\right) \\
\text { s.t. } & \mathbf{w}^{H} \mathbf{a}\left(\theta_{0}, r_{0}\right)=1 .
\end{aligned}
$$

The first term denotes the interference power minimization, and the second term stands for ensuring the target signal without distortion. The weight vector can be solved as

$$
\mathbf{w}_{\mathrm{MVDR}}=\frac{\mathbf{R}^{-\mathbf{1}} \mathbf{a}\left(\theta_{0}, r_{0}\right)}{\mathbf{a}^{H}\left(\theta_{0}, r_{0}\right) \mathbf{R a}\left(\theta_{0}, r_{0}\right)} .
$$

However, the MVDR beamformer does not make constraints on the sidelobe. In order to suppress the sidelobes, (17) can be reformulated as

$$
\begin{aligned}
\mathbf{w}=\underset{\mathbf{w}}{\arg \min } & \left(\mathbf{w}^{H} \mathbf{R w}\right) \\
\text { s.t. } & \mathbf{w}^{H} \mathbf{a}\left(\theta_{0}, r_{0}\right)=1 \\
& \left|\mathbf{w}^{H} \mathbf{a}\left(\theta_{\mathrm{SL}}, r_{\mathrm{SL}}\right)\right| \leq \varepsilon,
\end{aligned}
$$

where $\left(\theta_{\mathrm{SL}}, r_{\mathrm{SL}}\right)$ is the position of the sidelobe and $\varepsilon$ is the sidelobe level. Accordingly, the beampattern is given by

$$
P(\theta, r)=\left|\mathbf{a}^{H}(\theta, r) \mathbf{w}_{\mathrm{MVDR}}\right|^{2} .
$$

The SINR can be calculated by

$$
\operatorname{SINR}=\frac{\alpha_{0}^{2} \mathbf{w}^{H} \mathbf{a}\left(\theta_{0}, r_{0}\right) \mathbf{a}^{H}\left(\theta_{0}, r_{0}\right) \mathbf{w}}{\mathbf{w}^{H}\left(\sum_{i} \alpha_{i}^{2} \mathbf{a}\left(\theta_{i}, r_{i}\right) \mathbf{a}^{H}\left(\theta_{i}, r_{i}\right)+\mathbf{Q}\right) \mathbf{w}},
$$

where $\alpha_{0}^{2}$ and $\alpha_{i}^{2}$ are the target signal and interference signals averaged power, respectively, and the diagonal $\mathbf{Q}$ is the noise covariance matrix.

It is well known that inverse of an $M \times M$ Hermitian symmetric matrix requires $O\left(M^{3}\right)$ operations. For the FDA MIMO radar, since $\mathbf{y}$ is an $M N \times 1$ vector, computing $\mathbf{R}^{-1} \mathbf{a}\left(\theta_{0}, r_{0}\right)$ and $\mathbf{a}^{H}\left(\theta_{0}, r_{0}\right) \mathbf{R} \mathbf{a}\left(\theta_{0}, r_{0}\right)$ requires $O\left(M^{2} N^{2}+\right.$ $\left.M^{3} N^{3}\right)$ and $O\left(2 M^{3} N^{3}\right)$ operations, respectively [28]. Then, computation complexity is $O\left(M^{2} N^{2}+3 M^{3} N^{3}\right)$. The traditional MIMO radar has the same computation complexity. However, traditional MIMO radar has equal transmit and receiving array spacing, and its computing complexity will be $O\left((M+N)^{2}+3(M+N)^{3}\right)[29]$.

\section{FDA MIMO Radar Localization Performance and Resolution Probability Analysis}

In this section, we analyze the FDA MIMO radar localization performances in terms of CRLB and resolution probability. The FDA MIMO radar localization can estimate not only target angles, but also target ranges. We can regard both target and interferences as sources. In this case, (13) can be reformulated as

$$
\mathbf{y} \triangleq \operatorname{vec}(\mathbf{Y})=\sum_{s} \delta_{s}(\tau) \mathbf{a}\left(\theta_{s}, r_{s}\right)+\mathbf{n},
$$

where the subscript " $s$ " denotes the number index of targets. Defining $\mathbf{A}_{1}(\theta, r)=\mathbf{a}_{r} \mathbf{a}_{t r}^{T}$, we can get

$$
\mathbf{A}_{1}(\theta, r)
$$

$$
=\left[\begin{array}{cccc}
1 & e^{j \phi_{2}} & \cdots & e^{j(M-1) \phi_{2}} \\
e^{j \varphi} & e^{j \phi_{2}+j \varphi} & \cdots & e^{j(M-1) \phi_{2}+j \varphi} \\
e^{j 2 \varphi} & e^{j \phi_{2}+j 2 \varphi} & \cdots & e^{j(M-1) \phi_{2}+j 2 \varphi} \\
\vdots & \vdots & \cdots & \vdots \\
e^{j(N-1) \varphi} & e^{j \phi_{2}+j(N-1) \varphi} & \cdots & e^{j(M-1) \phi_{2}+j(N-1) \varphi}
\end{array}\right] .
$$


The first column is the receiving steering vector given in (9). Thus, we can estimate the target angle $\theta$ through the received signals. Similarly, the range $r$ can also be estimated. by

According to (22), the covariance matrix can be obtained

$$
\mathbf{R}_{y}=E\left\{\mathbf{y y}^{H}\right\}=\mathbf{A}_{2}(\theta, r) E\left[\boldsymbol{\delta}_{\mathbf{s}} \boldsymbol{\delta}_{\mathbf{s}}{ }^{H}\right] \mathbf{A}_{2}^{H}(\theta, r),
$$

where $\mathbf{A}_{2}(\theta, r)=\left[\mathbf{a}\left(\theta_{1}, r_{1}\right), \mathbf{a}\left(\theta_{2}, r_{2}\right), \ldots, \mathbf{a}\left(\theta_{S}, r_{S}\right)\right]$ is the receiving steering matrix for multiple signals with $S$ being the number of targets and $\boldsymbol{\delta}_{\mathbf{s}}=\left[\delta_{s}(1), \delta_{s}(2), \ldots, \delta_{s}(\tau)\right]$. For independent target signals and noise, $\mathbf{R}_{y}$ can be reformulated as

$$
\begin{aligned}
& \mathbf{R}_{y}=\mathbf{A}_{2}(\theta, r)\left(\begin{array}{ll}
\Lambda & 0 \\
0 & 0
\end{array}\right) \mathbf{A}_{2}(\theta, r)^{H}+\alpha_{n}^{2} \mathbf{I} \\
& =\left(\begin{array}{lll}
\mathbf{U}_{A} & \cdots & \mathbf{U}_{n}
\end{array}\right)\left(\begin{array}{cc}
\boldsymbol{\Lambda}+\sigma_{S}^{2} \mathbf{I} & 0 \\
0 & \sigma_{M-S}^{2} \mathbf{I}
\end{array}\right)\left(\begin{array}{lll}
\mathbf{U}_{A} & \cdots & \mathbf{U}_{n}
\end{array}\right)^{H},
\end{aligned}
$$

where $\mathbf{U}_{A}$ and $\mathbf{U}_{n}$ are the unitary matrices of signal and noise subspaces, respectively, $\boldsymbol{\Lambda}$ denotes the target signal power, and $\mathbf{I}$ is the unit matrix. The rank of $\Lambda+\sigma_{S}^{2} I$ is equal to the number of targets. Once the number of signals is obtained, the sizes of $\mathbf{U}_{A}$ and $\mathbf{U}_{n}$ are known accordingly. According to the MUSIC principle, the targets can be localized by searching the following peaks:

$$
P(\theta, r)=\frac{1}{\mathbf{a}^{H}(\theta, r) \mathbf{U}_{n} \mathbf{U}_{n}^{H} \mathbf{a}(\theta, r)} .
$$

The eigenvalue decomposition (EVD) of an $M \times M$ Hermitian symmetric matrix requires a computation complexity of $O\left(4(1 / 3) M^{3}\right)$ [28]. According to Section 3, the total computation complexity of an FDA MIMO radar is $O\left(5(1 / 3) M^{3} N^{3}\right)$, which is the same as that of a traditional MIMO radar. When the transmit and receiving arrays have the same element spacing, the computation complexity will be $O\left(5(1 / 3)(M+N)^{3}\right)[29]$.

To derive the CRLB, we rewrite (10) as

$$
\begin{aligned}
\mathbf{x}(l)= & \delta_{0} \mathbf{a}_{r}\left(\theta_{0}\right) \mathbf{a}_{t r}^{T}\left(\theta_{0}, r_{0}\right) \mathbf{s}(l) \\
& +\sum_{i} \delta_{i} \mathbf{a}_{r}\left(\theta_{i}\right) \mathbf{a}_{t r}^{T}\left(\theta_{i}, r_{i}\right) \mathbf{s}(l)+\mathbf{n}(l) \\
= & \sum_{s} \delta_{s} \mathbf{a}_{r}\left(\theta_{s}\right) \mathbf{a}_{t r}^{T}\left(\theta_{s}, r_{s}\right) \mathbf{s}(l)+\mathbf{n}(l) \\
= & \mathbf{B}(\theta) \mathbf{\Lambda} \mathbf{A}(\theta, r) \mathbf{s}(l)+\mathbf{n}(l)
\end{aligned}
$$

where

$$
\begin{aligned}
\mathbf{B}(\theta) & =\left[\begin{array}{llll}
\mathbf{a}_{r}\left(\theta_{1}\right) & \mathbf{a}_{r}\left(\theta_{2}\right) & \cdots & \mathbf{a}_{r}\left(\theta_{S}\right)
\end{array}\right], \\
\mathbf{A}(\theta, r) & =\left[\begin{array}{llll}
\mathbf{a}_{t r}\left(\theta_{1}, r_{1}\right) & \mathbf{a}_{t r}\left(\theta_{2}, r_{2}\right) & \cdots & \mathbf{a}_{t r}\left(\theta_{S}, r_{S}\right)
\end{array}\right], \\
\boldsymbol{\delta} & =\left[\begin{array}{llll}
\delta_{1} & \delta_{2} & \cdots & \delta_{S}
\end{array}\right], \\
\boldsymbol{\Lambda} & =\operatorname{diag}(\boldsymbol{\delta}),
\end{aligned}
$$

with diag denoting a diagonal matrix. Note that, for notation convenience, $\tau$ has been included in $\delta$. The unknown parameter vector to be estimated is then given by

$$
\boldsymbol{\eta}=\left[\begin{array}{llll}
\boldsymbol{\theta}^{T} & \mathbf{r}^{T} & \overline{\boldsymbol{\delta}}^{T} & \widehat{\boldsymbol{\delta}}^{T}
\end{array}\right]^{T},
$$

where $\boldsymbol{\theta}=\left[\theta_{1}, \theta_{2}, \ldots, \theta_{S}\right]^{T}, \mathbf{r}=\left[r_{1}, r_{2}, \ldots, r_{S}\right]^{T}, \overline{\boldsymbol{\delta}}=\left[\bar{\delta}_{1}\right.$, $\left.\bar{\delta}_{2}, \ldots, \bar{\delta}_{S}\right]^{T}$, and $\widehat{\delta}=\left[\widehat{\delta}_{1}, \widehat{\delta}_{2}, \ldots, \widehat{\delta}_{S}\right]^{T}$ are the real part and image part of $\boldsymbol{\delta}$, respectively. We divide $\boldsymbol{\eta}$ into deterministic and random components,

$$
\boldsymbol{\eta}=\left[\begin{array}{ll}
\boldsymbol{\beta}^{T} & \boldsymbol{\gamma}^{T}
\end{array}\right]^{T}
$$

where $\boldsymbol{\beta}=\left[\begin{array}{ll}\boldsymbol{\theta}^{T} & \mathbf{r}^{T}\end{array}\right]^{T}$ are the deterministic parameters and $\boldsymbol{\gamma}=$ $\left[\overline{\boldsymbol{\delta}}^{T}, \widehat{\boldsymbol{\delta}}^{T}\right]^{T}$ are random components. The Fisher information matrix (FIM) with respect to $\boldsymbol{\eta}$ is

$$
F\left(\beta_{i}, \beta_{j}\right)=2 \operatorname{Re}\left\{\operatorname{tr}\left[\frac{\partial\left(\mathbf{B}(\theta) \mathbf{\Lambda} \mathbf{A}^{T}(\theta, r) \mathbf{S}\right)^{H}}{\partial \beta_{i}} \mathbf{Q}^{-1} \frac{\partial\left(\mathbf{B}(\theta) \mathbf{\Lambda} \mathbf{A}^{T}(\theta, r) \mathbf{S}\right)}{\partial \beta_{j}}\right]\right\}
$$

where $\mathbf{Q}=\sigma^{2} \mathbf{I}$ is the noise covariance matrix, Re denotes the real part, and $\operatorname{tr}(\cdot)$ stands for the trace of a matrix. Equation (31) can be written as

$$
\begin{aligned}
& F\left(\beta_{i}, \beta_{j}\right)=\frac{2}{\sigma^{2}} \\
& \cdot \operatorname{Re}\left\{\operatorname{tr}\left[\frac{\partial\left(\mathbf{B}(\theta) \mathbf{\Lambda} \mathbf{A}^{T}(\theta, r)\right)}{\partial \beta_{j}} \mathbf{S S}^{H} \frac{\partial\left(\mathbf{B}(\theta) \mathbf{\Lambda} \mathbf{A}^{T}(\theta, r) \mathbf{S}\right)^{H}}{\partial \beta_{i}}\right]\right\} \\
& =\frac{2 L}{\sigma^{2}} \\
& \cdot \operatorname{Re}\left\{\operatorname{tr}\left[\frac{\partial\left(\mathbf{B}(\theta) \mathbf{\Lambda} \mathbf{A}^{T}(\theta, r)\right)}{\partial \beta_{i}} \frac{\partial\left(\mathbf{B}(\theta) \mathbf{\Lambda} \mathbf{A}^{T}(\theta, r) \mathbf{S}\right)^{H}}{\partial \beta_{j}}\right]\right\},
\end{aligned}
$$

where the facts that $\operatorname{tr}(\mathbf{A B})=\operatorname{tr}(\mathbf{B A})$ and $\mathbf{S S} S^{H} / L=\mathbf{I}$ are used. Due to the fact that $\operatorname{tr}(\mathbf{A B})=\operatorname{vec}\left(\mathbf{A}^{T}\right)^{T} \operatorname{vec}(\mathbf{B})^{T}$, we can get

$$
\begin{aligned}
& F\left(\beta_{i}, \beta_{j}\right)=\frac{2 L}{\sigma^{2}} \operatorname{Re}\left\{\operatorname{vec}\left[\frac{\partial\left(\mathbf{B}(\theta) \mathbf{\Lambda} \mathbf{A}^{T}(\theta, r)\right)^{H}}{\partial \beta_{i}}\right]^{H}\right. \\
& \left.\cdot \operatorname{vec}\left[\frac{\partial\left(\mathbf{B}(\theta) \mathbf{\Lambda} \mathbf{A}^{T}(\theta, r)\right)}{\partial \beta_{j}}\right]\right\} .
\end{aligned}
$$


We take a derivative with respect to $\theta_{i}$

$$
\begin{aligned}
\frac{\partial\left(\mathbf{B}(\theta) \Lambda \mathbf{A}^{T}(\theta, r)\right)}{\partial \theta_{i}}= & \dot{\mathbf{B}}(\theta) \mathbf{e}_{\mathbf{i}} \mathbf{e}_{\mathbf{i}}^{T} \boldsymbol{\Lambda} \mathbf{A}^{T}(\theta, r) \\
& +\mathbf{B}(\theta) \mathbf{e}_{\mathbf{i}} \mathbf{e}_{\mathbf{i}}^{T} \boldsymbol{\Lambda} \dot{\mathbf{A}}^{T}(\theta, r) \\
= & \frac{\partial \mathbf{a}_{r}\left(\theta_{i}\right)}{\partial \theta_{i}} \delta_{i} \mathbf{a}_{t r}\left(\theta_{i}, r_{i}\right)^{T} \\
& +\mathbf{a}_{r}\left(\theta_{i}\right) \delta_{i} \frac{\partial \mathbf{a}_{t r}\left(\theta_{i}, r_{i}\right)}{\partial \theta_{i}}
\end{aligned}
$$

where $\mathbf{e}_{\mathbf{i}}$ represents the $i$ th column of the unit matrix and $\dot{\mathbf{B}}$ denotes the derivation of $\mathbf{B}$ (similar for $\dot{\mathbf{A}}$ ). We take also a derivative with respect to $r_{i}$,

$$
\begin{aligned}
\frac{\partial\left(\mathbf{B}(\theta) \Lambda \mathbf{A}^{T}(\theta, r)\right)}{\partial r_{i}} & =\mathbf{B}(\theta) \mathbf{e}_{\mathbf{i}} \mathbf{e}_{\mathbf{i}}^{T} \boldsymbol{\Lambda} \dot{\mathbf{A}}^{T}(\theta, r) \\
& =\mathbf{a}_{r}\left(\theta_{i}\right) \delta_{i} \frac{\partial \mathbf{a}_{t r}\left(\theta_{i}, r_{i}\right)}{\partial r_{i}} .
\end{aligned}
$$

We then have

$$
\begin{aligned}
& \operatorname{vec}\left(\frac{\partial\left(\mathbf{B}(\theta) \boldsymbol{\Lambda} \mathbf{A}^{T}(\theta, r)\right)}{\partial \theta_{i}}\right) \\
& =\left[\frac{\partial \mathbf{a}_{r}\left(\theta_{i}\right)}{\partial \theta_{i}} \otimes \mathbf{a}_{t r}\left(\theta_{i}, r_{i}\right)+\mathbf{a}_{r}\left(\theta_{i}\right) \otimes \frac{\partial \mathbf{a}_{t r}\left(\theta_{i}, r_{i}\right)}{\partial \theta_{i}}\right] \delta_{i} \\
& =\mathbf{d}_{\theta}\left(\theta_{i}, r_{i}\right) \delta_{i} \\
& \operatorname{vec}\left(\frac{\partial\left(\mathbf{B}(\theta) \Lambda \mathbf{A}^{T}(\theta, r)\right)}{\partial r_{i}}\right) \\
& =\left[\mathbf{a}_{r}\left(\theta_{i}\right) \otimes \frac{\partial \mathbf{a}_{t r}\left(\theta_{i}, r_{i}\right)}{\partial r_{i}}\right] \delta_{i}=\mathbf{d}_{r}\left(\theta_{i}, r_{i}\right) \delta_{i},
\end{aligned}
$$

where the fact that $\operatorname{vec}\left(\mathbf{A V B}^{T}\right)=(\mathbf{B} \otimes \mathbf{A}) \operatorname{vec}(\mathbf{V})$ is utilized. Define

$$
\begin{aligned}
\Delta \triangleq & {\left[\mathbf{d}_{\theta}\left(\theta_{1}, r_{1}\right) \cdot \delta_{1}, \ldots, \mathbf{d}_{\theta}\left(\theta_{S}, r_{S}\right) \cdot \delta_{S} \mathbf{d}_{r}\left(\theta_{1}, r_{1}\right)\right.} \\
& \left.\cdot \delta_{1}, \ldots, \mathbf{d}_{r}\left(\theta_{S}, r_{S}\right) \cdot \delta_{S}\right] .
\end{aligned}
$$

The FIM with respect to $\boldsymbol{\beta}$ part can be calculated by

$$
\mathbf{F}(\boldsymbol{\beta}, \boldsymbol{\beta})=\frac{2 L}{\sigma^{2}} \operatorname{Re}\left\{\Delta^{H} \cdot \Delta\right\} .
$$

Similarly, we have

$$
\begin{aligned}
& \operatorname{vec}\left(\frac{\partial\left(\mathbf{B}(\theta) \mathbf{\Lambda} \mathbf{A}^{T}(\theta, r)\right)}{\partial \bar{\delta}_{i}}\right)=\mathbf{a}_{r}\left(\theta_{i}\right) \otimes \mathbf{a}_{t r}\left(\theta_{i}, r_{i}\right), \\
& \operatorname{vec}\left(\frac{\partial\left(\mathbf{B}(\theta) \Lambda \mathbf{A}^{T}(\theta, r)\right)}{\partial \hat{\delta}_{i}}\right)=j \mathbf{a}_{r}\left(\theta_{i}\right) \otimes \mathbf{a}_{t r}\left(\theta_{i}, r_{i}\right),
\end{aligned}
$$

where $\bar{\delta}_{i}$ and $\widehat{\delta}_{i}$ are the real and imaginary part of reflection coefficient $\delta_{i}$, respectively. Defining $\mathbf{v}_{i}=\mathbf{a}_{r}\left(\theta_{i}\right) \otimes \mathbf{a}_{t r}\left(\theta_{i}, r_{i}\right)$ and $\mathbf{V}=\left[\mathbf{v}_{\mathbf{1}}, \mathbf{v}_{\mathbf{2}}, \ldots, \mathbf{v}_{\mathbf{S}}\right]$, we can get $[30]$

$$
\begin{aligned}
& \mathbf{F}(\beta, \bar{\delta})=\frac{2 L}{\sigma^{2}} \operatorname{Re}\left\{\Delta^{H} \cdot \mathbf{V}\right\} \\
& \mathbf{F}(\beta, \widehat{\delta})=-\frac{2 L}{\sigma^{2}} \operatorname{Im}\left\{\Delta^{H} \cdot \mathbf{V}\right\} \\
& \mathbf{F}(\bar{\delta}, \bar{\delta})=\mathbf{F}(\widehat{\delta}, \widehat{\delta})=\frac{2 L}{\sigma^{2}} \operatorname{Re}\left\{\mathbf{V}^{H} \cdot \mathbf{V}\right\}
\end{aligned}
$$

Therefore, the FIM of FDA MIMO radar is derived as

$$
\begin{aligned}
& \mathbf{F}=\left[\begin{array}{lll}
\mathbf{F}(\beta, \beta) & \mathbf{F}(\beta, \bar{\delta}) & \mathbf{F}(\beta, \widehat{\delta}) \\
\mathbf{F}(\beta, \bar{\delta}) & \mathbf{F}(\bar{\delta}, \bar{\delta}) & \mathbf{F}(\bar{\delta}, \widehat{\delta}) \\
\mathbf{F}(\widehat{\delta}, \beta) & \mathbf{F}(\widehat{\alpha}, \bar{\delta}) & \mathbf{F}(\widehat{\delta}, \widehat{\delta})
\end{array}\right] \\
&= \frac{2 L}{\sigma^{2}}\left[\begin{array}{lll}
\operatorname{Re}\left\{\Delta^{H} \Delta\right\} & \operatorname{Re}\left\{\Delta^{H} \mathbf{V}\right\} & -\operatorname{Im}\left\{\Delta^{H} \mathbf{V}\right\} \\
\operatorname{Re}\left\{\Delta^{H} \mathbf{V}\right\} & \operatorname{Re}\left\{\mathbf{V}^{H} \mathbf{V}\right\} & -\operatorname{Im}\left\{\mathbf{V}^{H} \mathbf{V}\right\} \\
\operatorname{Im}\left\{\Delta^{H} \mathbf{V}\right\} & \operatorname{Im}\left\{\mathbf{V}^{H} \mathbf{V}\right\} & \operatorname{Re}\left\{\mathbf{V}^{H} \mathbf{V}\right\}
\end{array}\right] \\
&= \frac{2 L}{\sigma^{2}} \operatorname{Re}\left[\begin{array}{ccc}
\Delta^{H} \Delta & \Delta^{H} \mathbf{V} & -j \Delta^{H} \mathbf{V} \\
\Delta^{H} \mathbf{V} & \mathbf{V}^{H} \mathbf{V} & -j \mathbf{V}^{H} \mathbf{V} \\
j \Delta^{H} \mathbf{V} & j \mathbf{V}^{H} \mathbf{V} & \mathbf{V}^{H} \mathbf{V}
\end{array}\right] \\
&= \frac{2 L}{\sigma^{2}} \operatorname{Re}\left\{\left[\begin{array}{lll}
\Delta^{H} \\
\mathbf{V}^{H} \\
j \mathbf{V}^{H}
\end{array}\right]\right. \\
& {\left[\begin{array}{lll}
\Delta & \mathbf{V} & -j \mathbf{V}]\}
\end{array}\right] }
\end{aligned}
$$

Finally, the CRLBs with respect to $\theta_{i}$ and $r_{i}$ are the two diagonal elements of the inverse of the FIM:

$$
\begin{aligned}
\mathrm{CRLB}_{\theta i} & =\left[\mathbf{F}^{-1}\right]_{i, i} \\
\mathrm{CRLB}_{r i} & =\left[\mathbf{F}^{-1}\right]_{S+i, S+i},
\end{aligned}
$$

where $\mathbf{F}^{-1}$ is the inverse of $\mathbf{F}$ and $[\cdot]_{i, j}$ is the element at the $i$ th row and $j$ th column of the matrix. Since matrices $\Delta$ and $\mathbf{V}$ depend on both range and $\Delta f$, the CRLBs are influenced by $\Delta f$.

Furthermore, we use the resolution probability defined as follows to compare the localization performance. Two targets can be resolved only when the following relation is achieved:

$$
\begin{aligned}
& \left|\hat{\theta}_{1}-\theta_{1}\right| \leq\left|\frac{\theta_{1}-\theta_{2}}{2}\right| \\
& \left|\hat{\theta}_{2}-\theta_{2}\right| \leq\left|\frac{\theta_{1}-\theta_{2}}{2}\right|,
\end{aligned}
$$

where $\theta_{1}$ and $\theta_{2}$ are the true directions. 


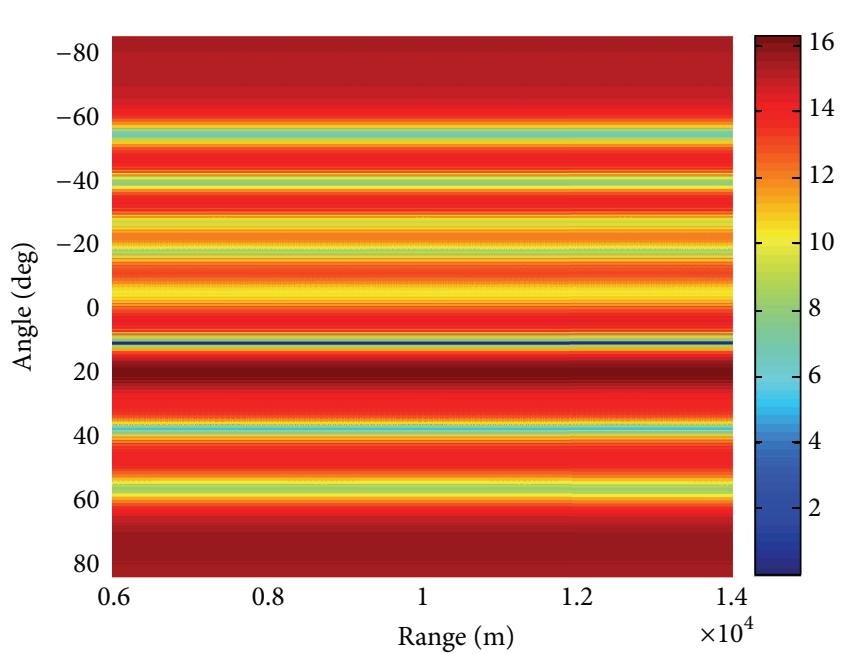

(a)

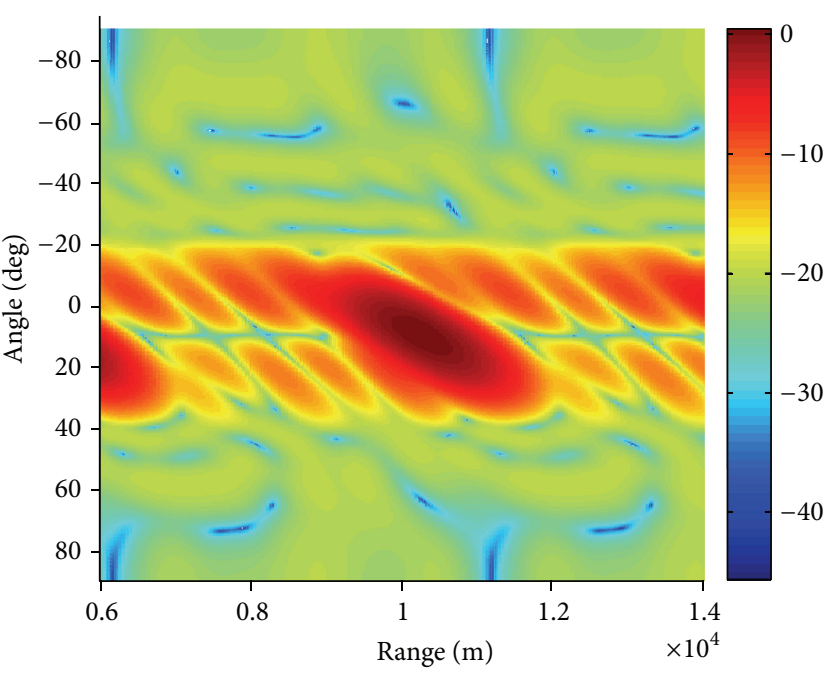

(b)

FIGURE 1: Comparative transmit beampattern: (a) conventional MIMO radar and (b) FDA MIMO radar.

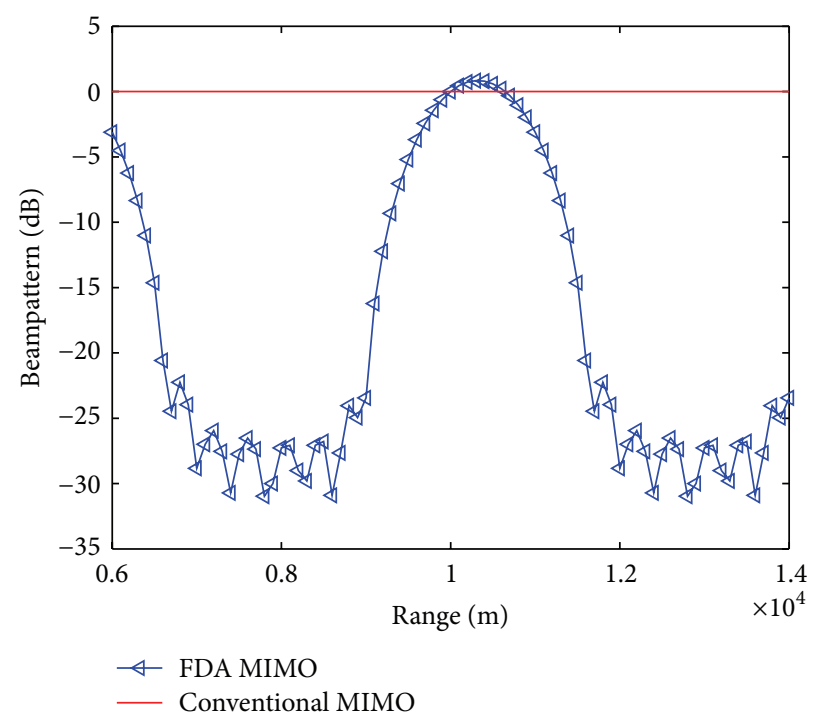

FIGURE 2: Comparative transmit beampattern in range profile $\theta=10^{\circ}$.

\section{Simulation Results}

Suppose the following simulation parameters: $M=N=8$, $f_{0}=10 \mathrm{GHz}, d=\lambda / 2, c=3 \times 10^{8} \mathrm{~m} / \mathrm{s}, \Delta f=30 \mathrm{KHz}, L=256$, SINR $=10 \mathrm{~dB}$, and $\tau=256$. The additive noise is modeled as complex Gaussian zero-mean spatially and temporally white random sequences.

Example 1 (adaptive beamforming and performance analysis). In the first example, one target of interest and multiple interferences are located at $\left(\theta_{s}, r_{s}\right)=\left(10^{\circ}, 10 \mathrm{~km}\right)$ and $\left(\theta_{j}, r_{j}\right)$ $=\left(10^{\circ}, 6.5 \sim 9 \mathrm{~km}\right)$, respectively.

Figure 1(a) gives the adaptive beampattern of conventional MIMO radar. Obviously, it cannot suppress the interferences having the same angle as the targets shown in
Figure 2. In this case, the interferences will degrade the SINR performance. In contrast, the beampattern mainlobe of FDA MIMO radar can be steered to suppress range-dependent interferences, as shown in Figure 1(b) and Figure 2. The FDA MIMO radar beampattern has zero nullings at the interference locations and, thus, the sidelobes are significantly suppressed.

We also examined the adaptive beamformer output SINR versus input SINR. In the first case, we assume that the target and interference have the same angle but different ranges. Figure 3(a) shows the comparative beamforming performance. It is noticed that FDA MIMO radar has a much higher output SINR than conventional MIMO radar. In the second case, the target and interference are at angles $\theta_{s}=$ $10^{\circ}$ and $\theta_{i}=-10^{\circ}$, respectively. It is seen from Figure 3(b) 


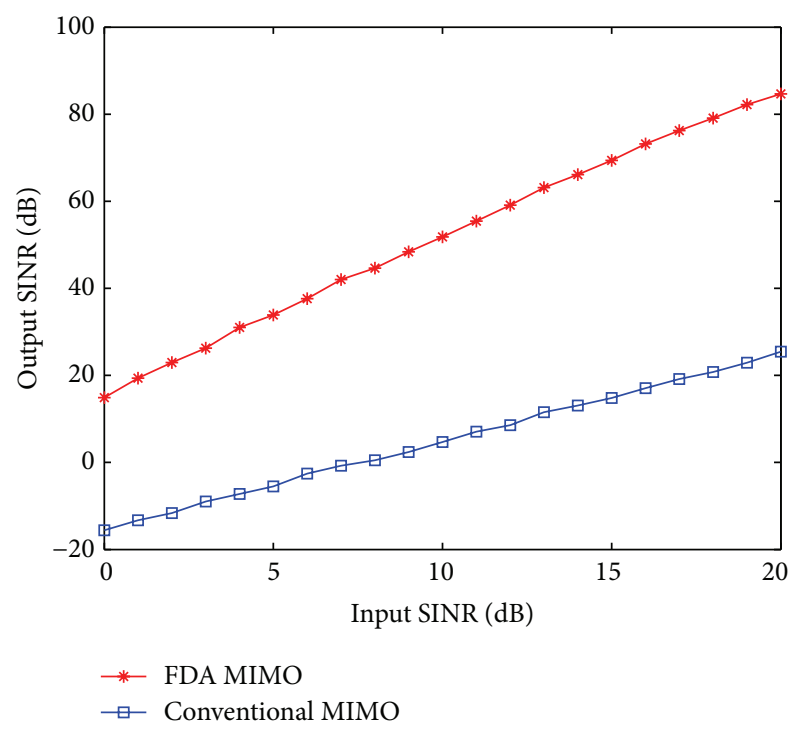

(a)

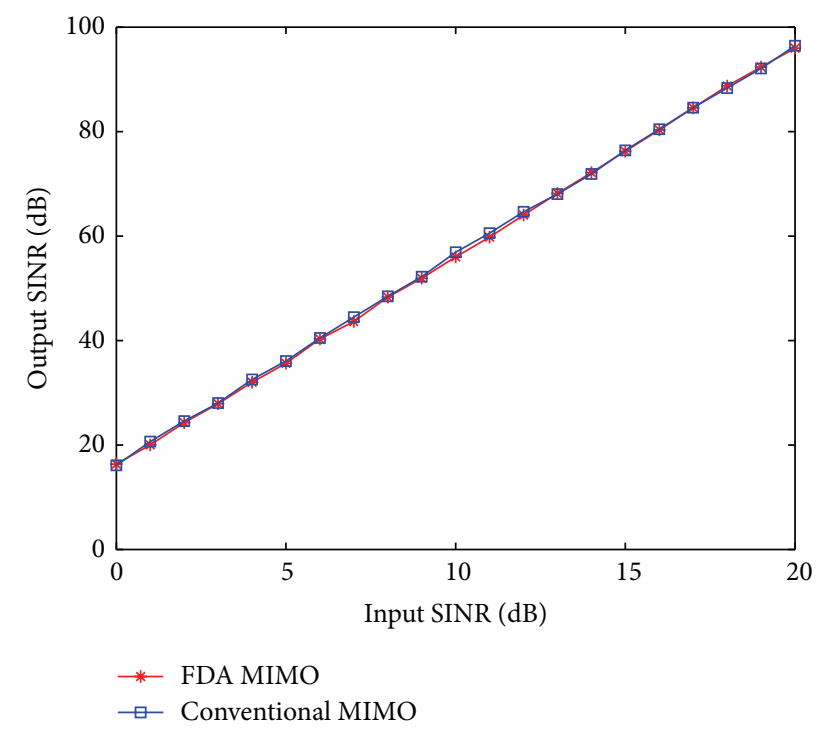

(b)

FIGURE 3: Output SINR comparisons: (a) target and interferences are at the same angle but different ranges; (b) target and interferences are at different angles.

that the output SINR of FDA MIMO radar is equivalent to that of phased MIMO radar. Consequently, FDA MIMO radar outperforms conventional MIMO radar in interference suppression.

Example 2 (localization and performance analysis). Consider four targets with locations $\left(-20^{\circ}, 8 \mathrm{~km}\right),\left(20^{\circ}, 8 \mathrm{~km}\right),\left(-20^{\circ}\right.$, $10 \mathrm{~km})$, and $\left(20^{\circ}, 10 \mathrm{~km}\right)$ and $\mathrm{SNR}=10 \mathrm{~dB}$. Figure 4 compares the localization results. Since conventional MIMO radar can only estimate target angles, there are only two curves for conventional MIMO radar. Consequently, conventional MIMO radar fails to resolve the four targets completely.

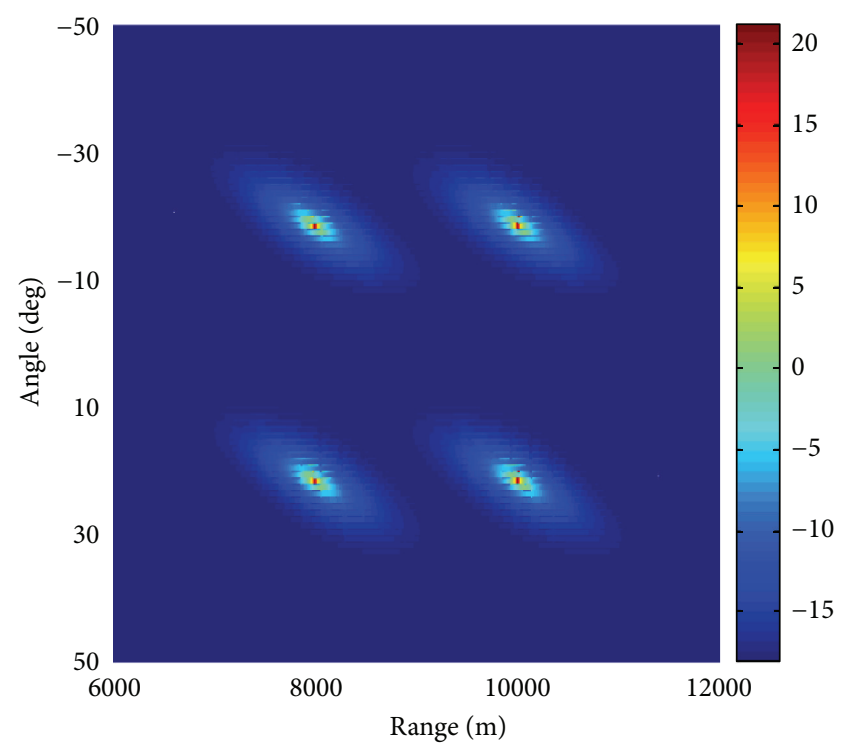

(a)

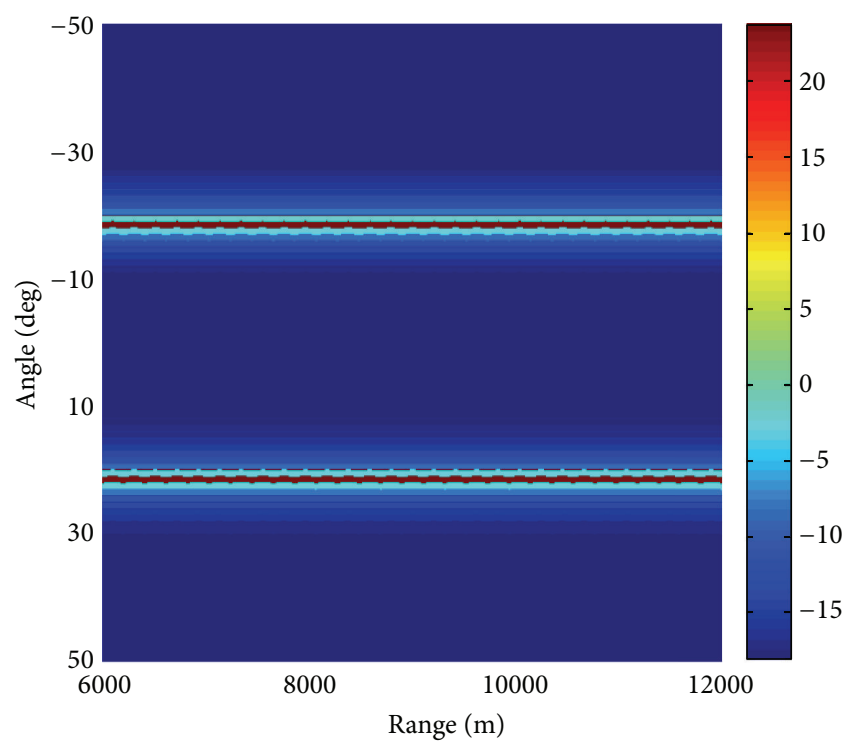

(b)

FIGURE 4: Power spectra: (a) FDA MIMO radar and (b) conventional MIMO radar.

In contrast, the four targets can be easily identified by the FDA MIMO radar, as shown in Figure 4(a). Furthermore, to clearly compare the performance, the amplitude spectrum profile cut at angle $20^{\circ}$ is given in Figure 5. It shows that the target ranges are correctly estimated by the FDA MIMO radar.

Figure 6 compares the CRLB as a function of SNR. The FDA MIMO radar steering vector depends on $\Delta f$. Figure $6(\mathrm{a})$ gives the CRLB for angle $\theta$ estimation at different $\Delta f$. It is seen that CRLB for $\theta$ holds as $\Delta f$ increases, whereas CRLB for range $r$ decreases as $\Delta f$ increases, as shown in Figure 6(b).

Figures 7(a) and 7(b) show the mean square error (MSE) [31] and CRLB on angle and range estimations versus SNR, respectively. Note that the MSEs are computed based on 100 


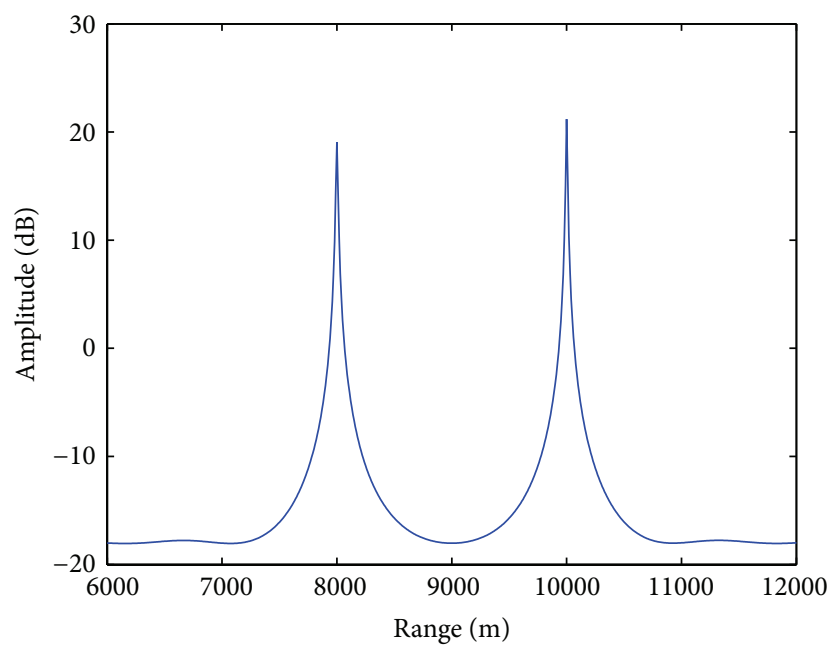

FIgURE 5: Power spectra profile for FDA MIMO radar in range dimension for angle $20^{\circ}$.

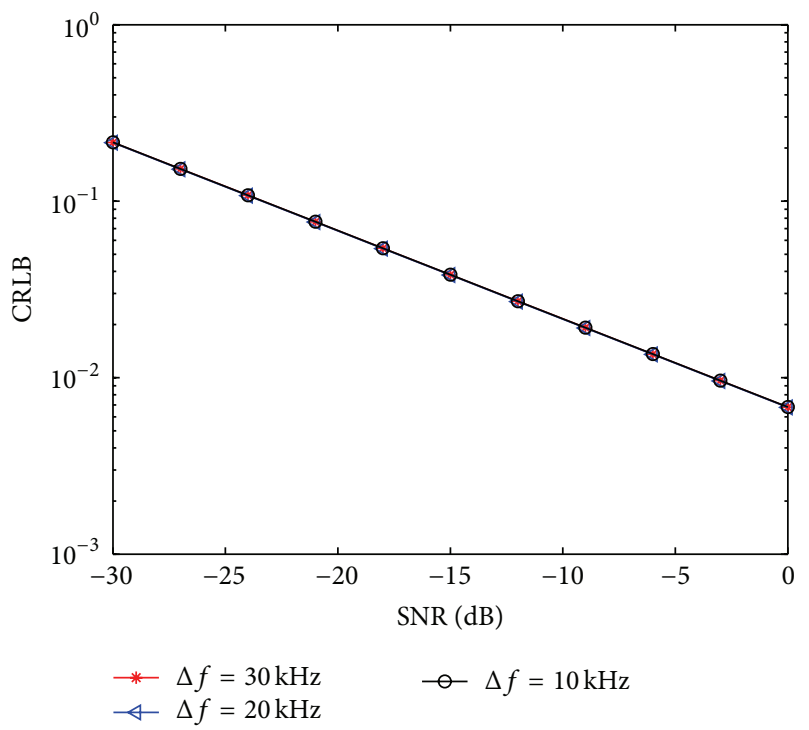

(a)

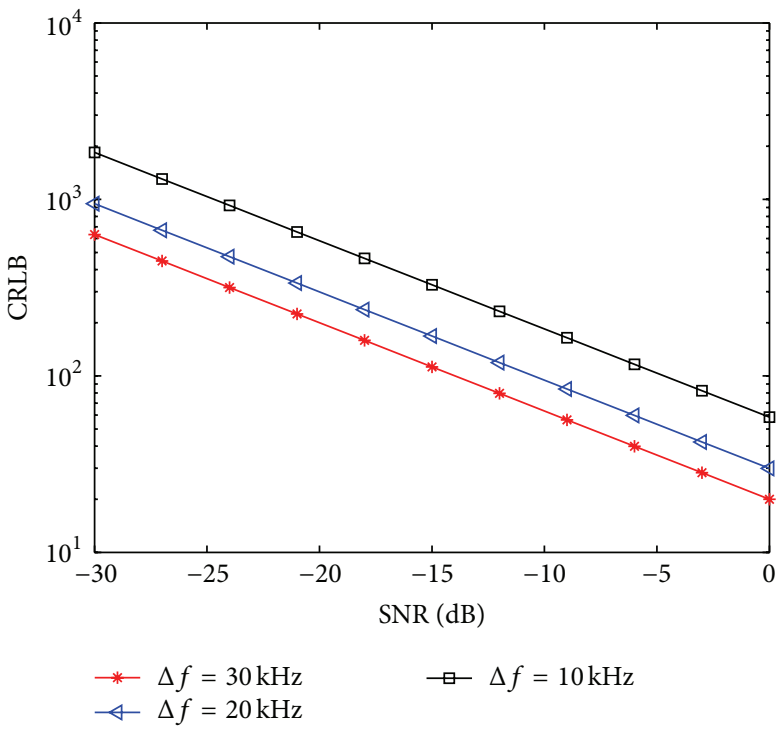

(b)

FIGURE 6: Comparative CRLB results for FDA MIMO radar with different $\Delta f$ : (a) angle estimation and (b) range estimation.

independent Monte Carlo simulation runs. In Figure 7(a), the conventional MIMO radar has two target signals having the same angle whereas the FDA MIMO radar MSE is obtained by considering only one target. Therefore, the previous result outperforms that of the FDA MIMO radar in angle estimation. Nevertheless, it can be seen that the proposed approach gives a satisfactory estimation performance.

Finally, the resolution probability performances are compared in Figure 8(a). It can be seen that FDA MIMO radar still outperforms conventional MIMO radar. Figure 8(b) shows the resolution probability versus $\Delta f$. Since $\Delta f r=c_{0}$, if $\Delta f$ increases, the range $r$ will decrease. Hence, the resolution probability will decrease along with the decrease of $\Delta f$ for FDA MIMO radar.

\section{Conclusion}

In this paper, we proposed an FDA MIMO adaptive beamforming and localization scheme for range-dependent targets and interferences with the same angle but different ranges. The FDA MIMO radar provides a promising rangedependent beampattern, which is particularly valuable for suppressing range-dependent interferences. The FDA MIMO radar has higher output SINR than the conventional MIMO radar. Besides, the ranges and angles of targets can be solely estimated with MUSIC-based algorithm. This conclusion is also validated by the CRLB and MSE for FDA MIMO radar which are also analyzed to examine the estimation performance comparisons. 


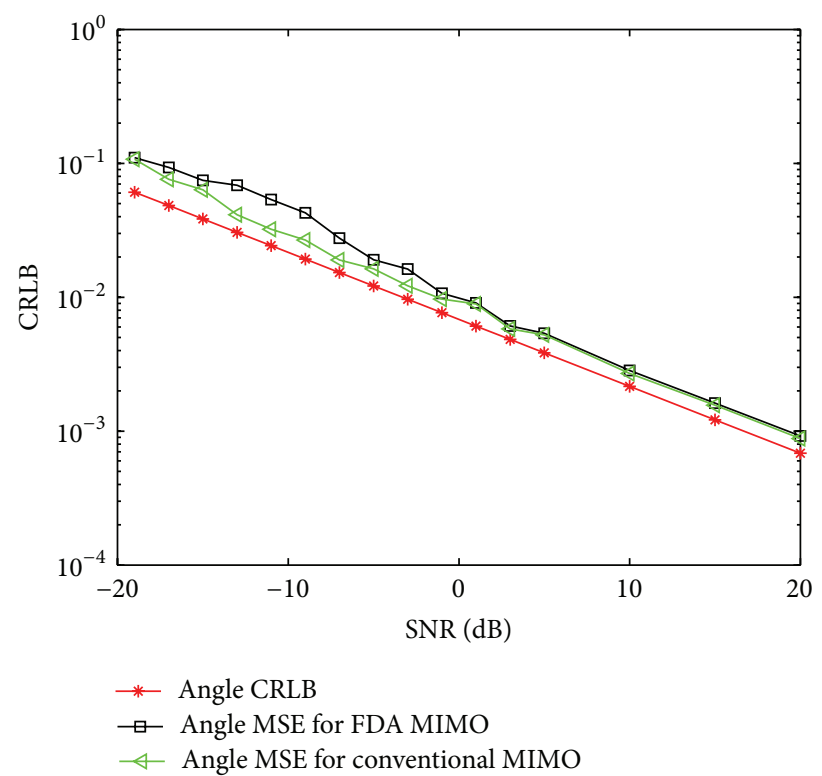

(a)

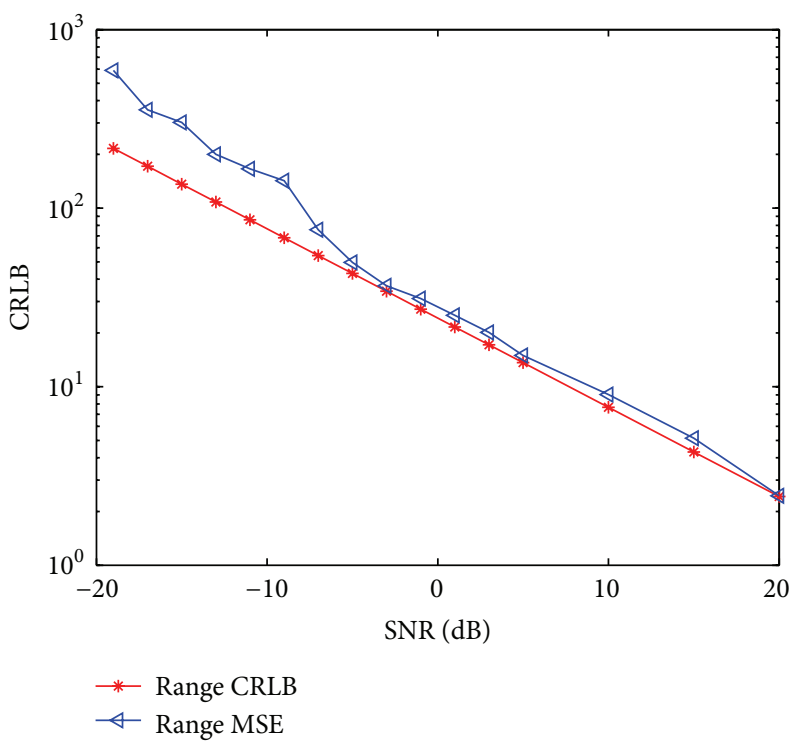

(b)

FIGURE 7: Comparison of CRLB and MSE for FDA MIMO radar: (a) angle estimation and (b) range estimation.

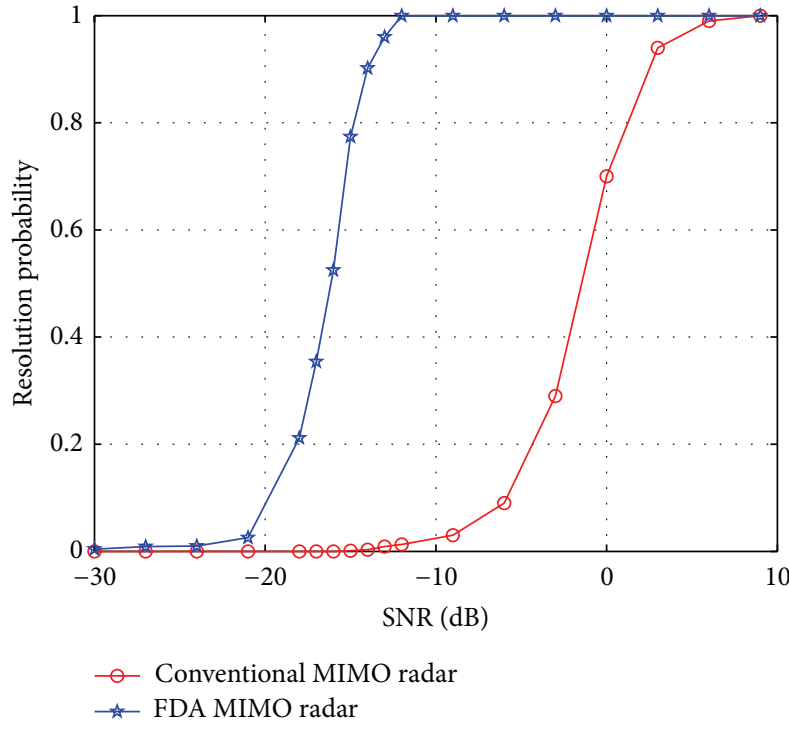

(a)

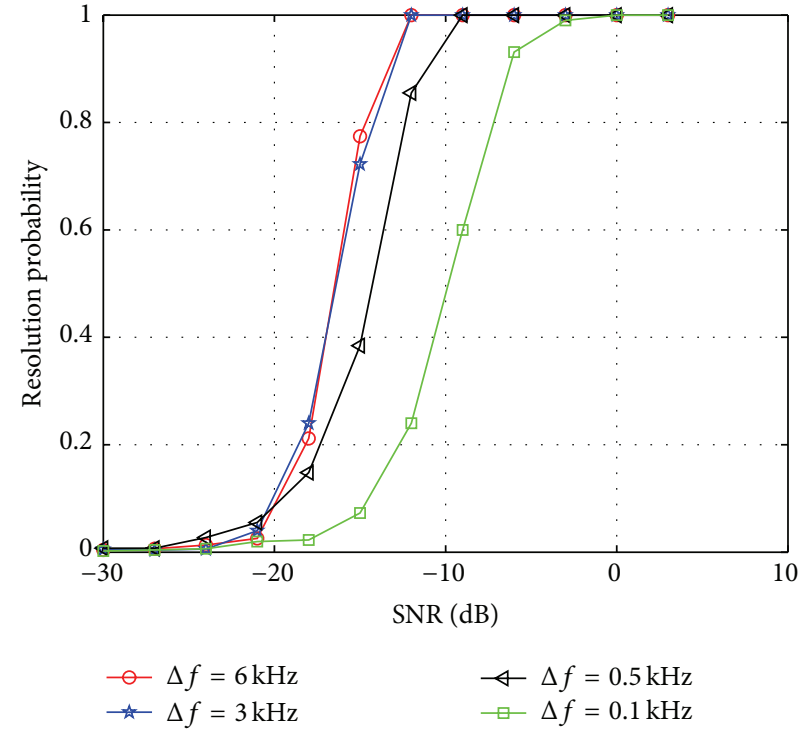

(b)

FIGURE 8: Resolution probability: (a) FDA MIMO radar and conventional MIMO radar and (b) different $\Delta f$ for FDA MIMO radar.

\section{Conflict of Interests}

The authors declare that there is no conflict of interests regarding the publication of this paper.

\section{Acknowledgment}

This work was supported in part by the Program for New Century Excellent.

\section{References}

[1] B. D. Van Veen and K. M. Buckley, "Beamforming: a versatile approach to spatial filtering," IEEE ASSP Magazine, vol. 5, no. 2, pp. 4-24, 1988.

[2] E. Tuncer and B. Freidlander, Classical and Modern Directionof-Arrival Estimation, Elsevier, New York, NY, USA, 2009.

[3] J. Ding, H. Chen, H. Wang, X. Li, and Z. Zhuang, "Low-grazing angle detection in compound-gaussian clutter with hybrid 
MIMO radar," International Journal of Antennas and Propagation, vol. 2013, Article ID 374342, 8 pages, 2013.

[4] Y. A. S. Dama, R. A. Abd-Alhameed, S. M. R. Jones et al., "An envelope correlation formula for (N, N) MIMO antenna arrays using input scattering parameters, and including power losses," International Journal of Antennas and Propagation, vol. 2011, Article ID 421691, 7 pages, 2011.

[5] C. Yunhe, Z. Zijing, W. Shenghua, and D. Fengzhou, "Direction finding for bistatic MIMO radar with uniform circular array," International Journal of Antennas and Propagation, vol. 2013, Article ID 674878, 6 pages, 2013.

[6] P. Antonik, M. C. Wicks, H. D. Griffiths, and C. J. Baker, "Frequency diverse array radars," in Proceedings of the IEEE Radar Conference, pp. 215-217, Verona, NY, USA, April 2006.

[7] M. C. Wicks and P. Antonik, "Frequency diverse array with independent modulation of frequency, amplitude, and phase," U.S.A Patent 7,319,427, January 2008.

[8] P. Antonik and M. C. Wicks, "Method and apparatus for simultaneous synthetic aperture and moving target indication," U.S.A Patent, application 20080129584, June 2008.

[9] P. Antonik, M. C. Wicks, H. D. Griffiths, and C. J. Baker, "Multimission multi-mode waveform diversity," in Proceedings of the IEEE Radar Conference, pp. 580-582, IEEE, Verona, NY, USA, April 2006.

[10] P. Antonik and M. C. Wicks, "Method and apparatus for a frequency diverse array," U.S.A Patent 7.511,665B2, March 2009.

[11] P. Antonik, An investigation of a frequency diverse array [Ph.D. dissertation], University College London, London, UK, 2009.

[12] A. Aytun, Frequency diverse array radar [M.S. thesis], Naval Postgraduate School, 2010.

[13] S. Brady, Frequency diverse array radar: signal characterization and measurement accuracy [M.S. thesis], Air Force Institute of Technology, 2010.

[14] M. Secmen, S. Demir, A. Hizal, and T. Eker, "Frequency diverse array antenna with periodic time modulated pattern in range and angle," in Proceedings of the IEEE Radar Conference, pp. 427-430, Boston, Mass, USA, April 2007.

[15] P. Baizert, T. B. Hale, M. A. Temple, and M. C. Wicks, "Forwardlooking radar GMTI benefits using a linear frequency diverse array," Electronics Letters, vol. 42, no. 22, pp. 1311-1312, 2006.

[16] P. F. Sammartino and C. J. Baker, "The frequency diverse bistatic system," in Proceedings of the International Waveform Diversity and Design Conference (WDD '09), pp. 155-159, Orlando, Fla, USA, February 2009.

[17] J. Farooq, M. A. Temple, and M. A. Saville, "Application of frequency diverse arrays to synthetic aperture radar imaging," in Proceedings of the International Conference on Electromagnetics in Advanced Applications (ICEAA '07), pp. 447-449, Torino, Italy, September 2007.

[18] J. Farooq, M. A. Temple, and M. A. Saville, "Exploiting frequency diverse array processing to improve SAR image resolution," in Proceedings of the IEEE Radar Conference (RADAR '08), pp. 1-5, IEEE, Rome, Italy, May 2008.

[19] J. Farooq, Frequency diversity for improving synthetic aperture radar imaging [Ph.D. thesis], Air Force Institute of Technology, 2009.

[20] Y. Wang, W.-Q. Wang, and H. Shao, "Frequency diverse array radar cramér-rao lower bounds for estimating direction, range, and velocity," International Journal of Antennas and Propagation, vol. 2014, Article ID 830869, 10 pages, 2014.
[21] V. Ravenni, "Performance evaluations of frequency diversity radar system," in Proceedings of the 4th European Radar Conference, pp. 436-439, Munich, Germany, October 2007.

[22] W.-Q. Wang, "Range-angle dependent transmit beampattern synthesis for linear frequency diverse arrays," IEEE Transactions on Antennas and Propagation, vol. 61, no. 8, pp. 4073-4081, 2013.

[23] P. F. Sammartino, C. J. Baker, and H. D. Griffiths, "Frequency diverse MIMO techniques for radar," IEEE Transactions on Aerospace and Electronic Systems, vol. 49, no. 1, pp. 201-222, 2013.

[24] L. Zhuang and X. Z. Liu, "Application of frequency diversity to suppress grating lobes in coherent MIMO radar with separated subapertures," EURASIP Journal on Advances in Signal Processing, vol. 2009, Article ID 481792, 10 pages, 2009.

[25] W.-Q. Wang, "Phased-MIMO radar with frequency diversity for range-dependent beamforming," IEEE Sensors Journal, vol. 13, no. 4, pp. 1320-1328, 2013.

[26] W.-Q. Wang and H. Shao, "Range-angle localization of targets by a double-pulse frequency diverse array radar," IEEE Journal on Selected Topics in Signal Processing, vol. 8, no. 1, pp. 106-114, 2013.

[27] W.-Q. Wang and H. C. So, “Transmit subaperturing for range and angle estimation in frequency diverse array radar," IEEE Transactions on Signal Processing, vol. 62, no. 8, pp. 2000-2011, 2014.

[28] S. D. Somasundaram, N. H. Parsons, P. Li, and R. C. de Lamare, "Reduced-dimension robust capon beamforming using Krylovsubspace techniques," IEEE Transactions on Aerospace and Electronic Systems, vol. 51, no. 1, pp. 270-289, 2015.

[29] K. W. Forsythe, D. W. Bliss, and G. S. Fawcett, "Multipleinput multiple-output (MIMO) radar: performance issues," in Proceedings of the 38th Asilomar Conference on Signals, Systems and Computers, vol. 1, pp. 310-315, IEEE, Pacific Grove, Calif, USA, November 2004.

[30] J. Li, L. Xu, P. Stoica, K. W. Forsythe, and D. W. Bliss, "Range compression and waveform optimization for MIMO radar: a Cramér-Rao bound based study," IEEE Transactions on Signal Processing, vol. 56, no. 1, pp. 218-232, 2008.

[31] L. W. Nolte, S. C. Lee, and S.-C. Liu, "On performance of array signal processing," IEEE Journal of Oceanic Engineering, vol. 12, no. 1, pp. 148-154, 1987. 

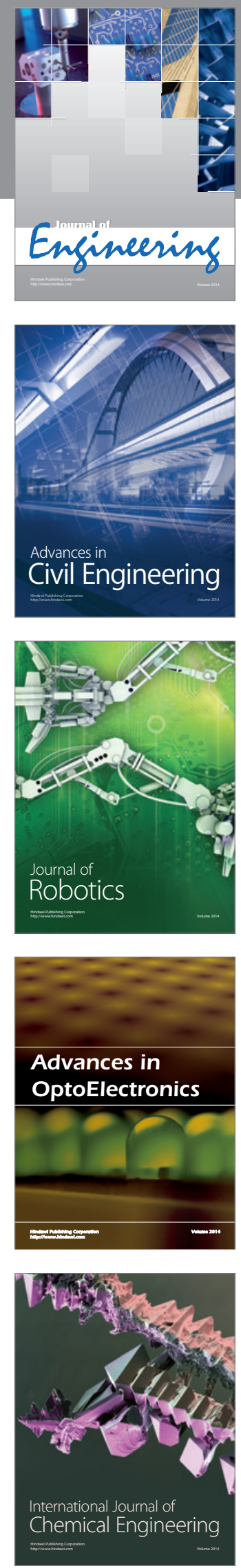

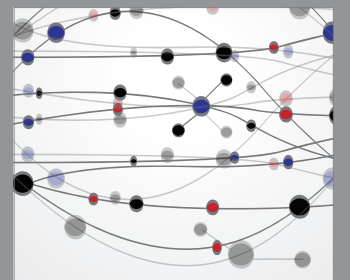

The Scientific World Journal
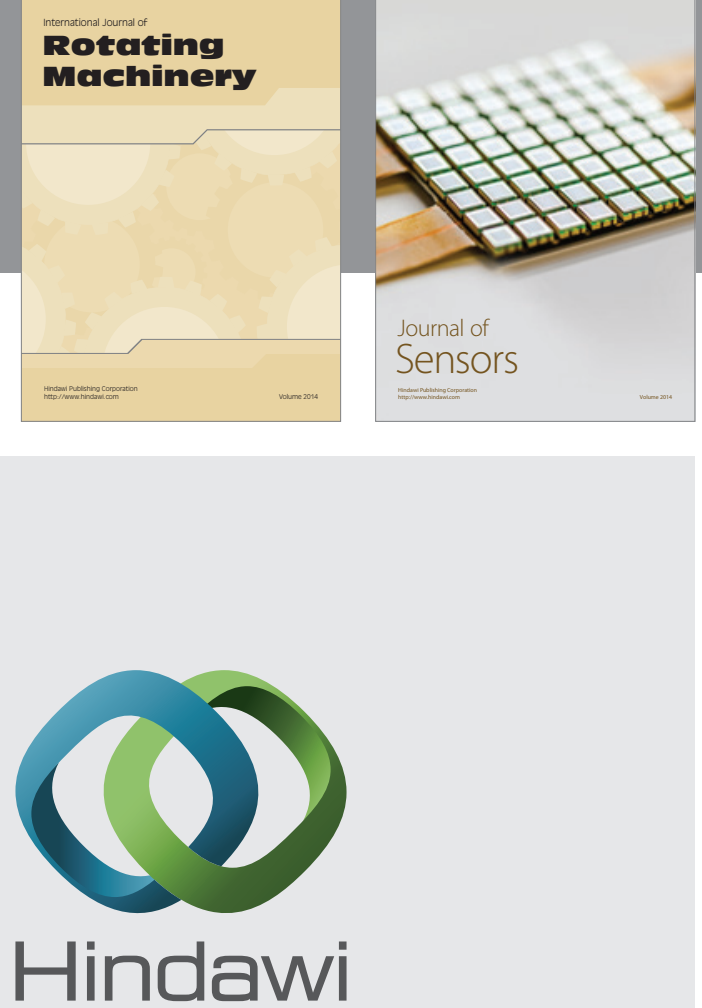

Submit your manuscripts at http://www.hindawi.com
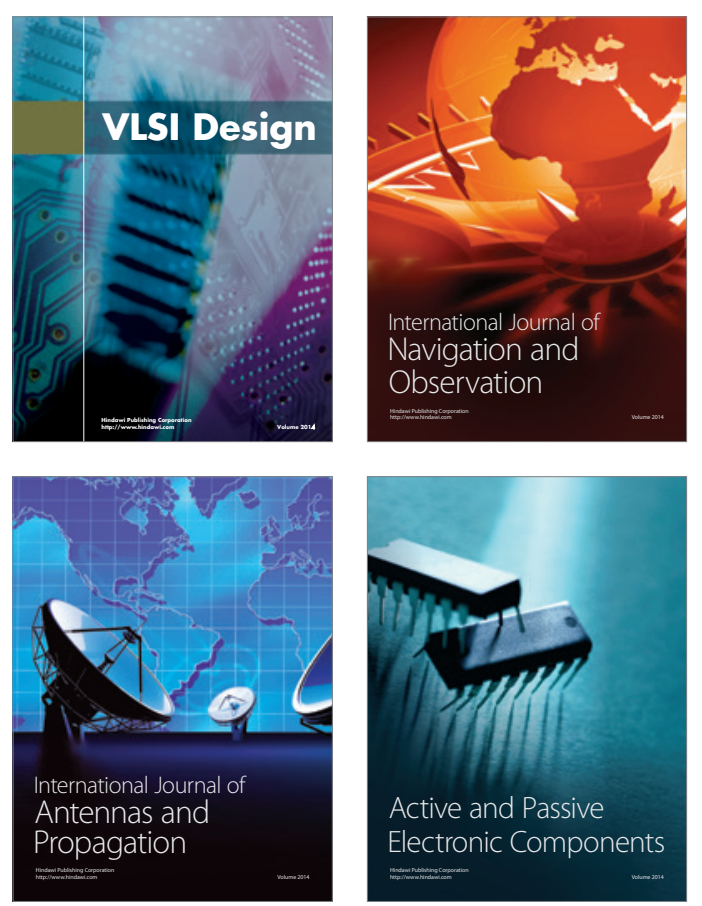
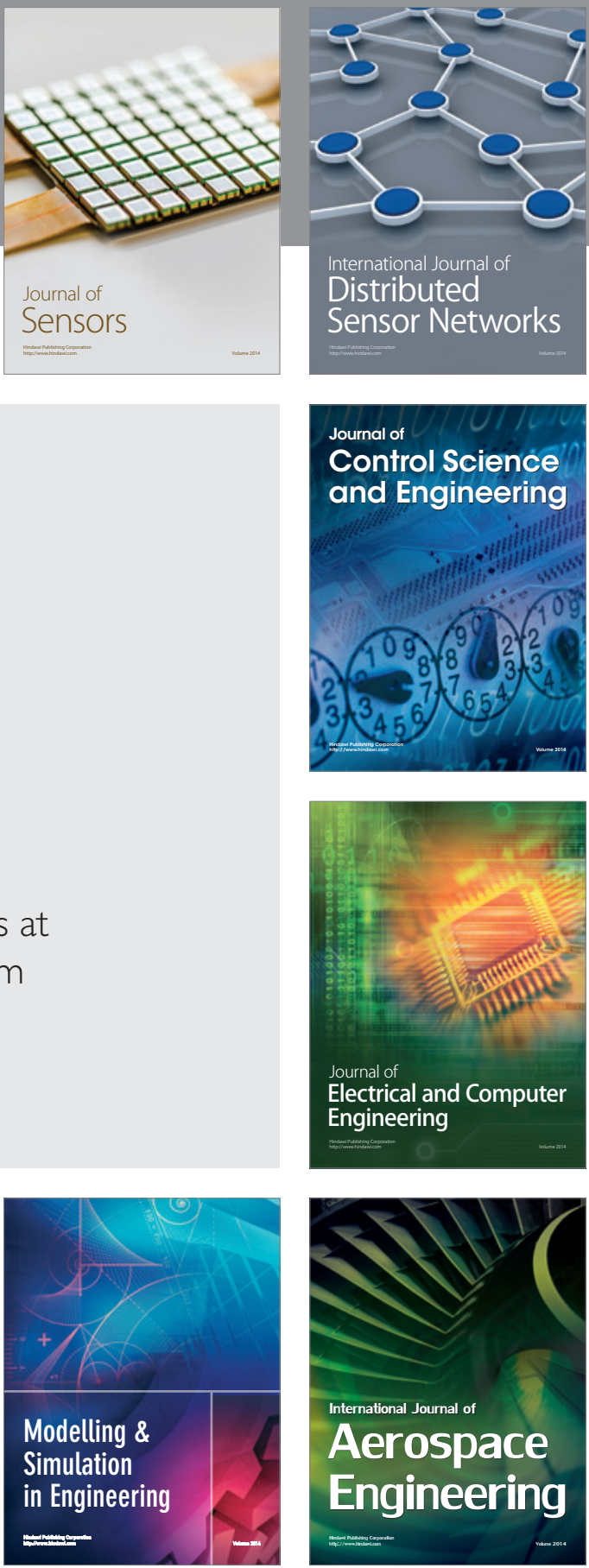

Journal of

Control Science

and Engineering
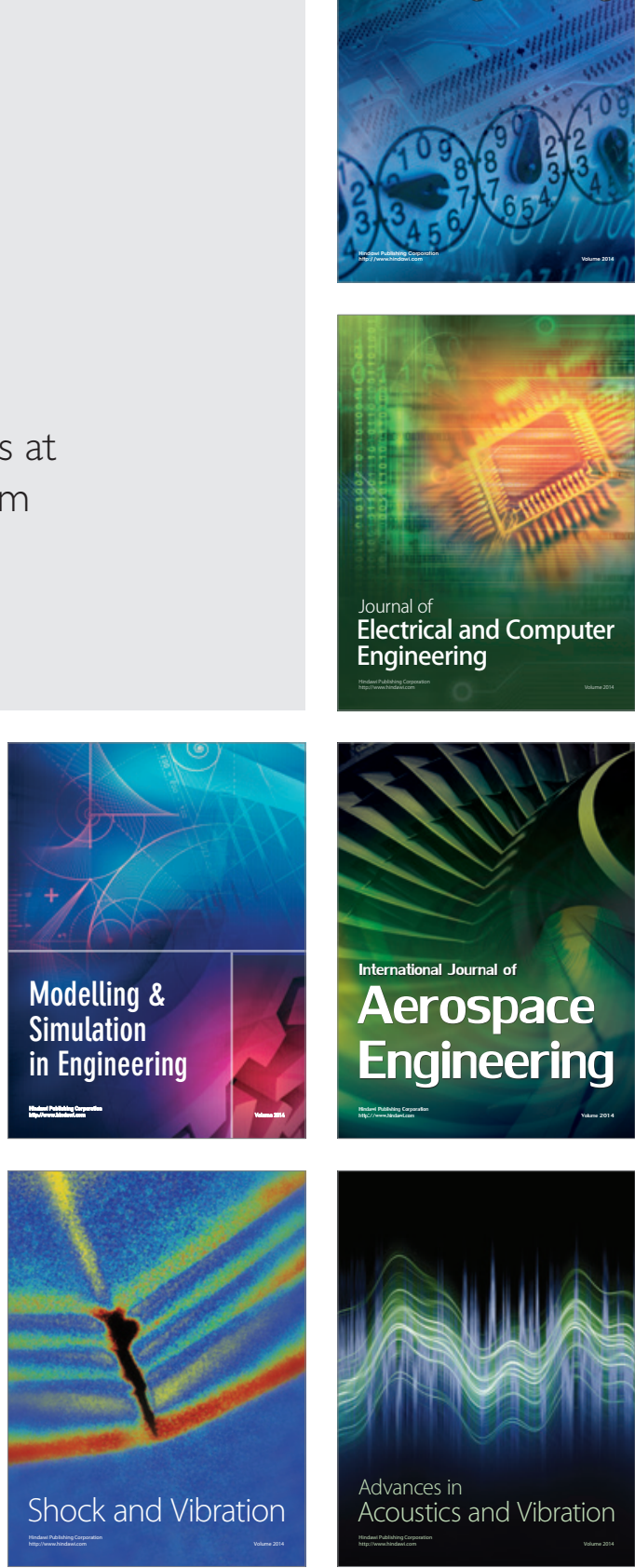